\title{
The validity of the Community Periodontal Index of Treatment Needs (CPITN) in epidemiological studies of periodontal diseases
}

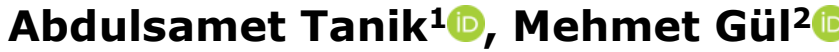 \\ ${ }^{1}$ Adıyaman University, Faculty of Dentistry, Department of Periodontology, Adıyaman, Turkey \\ ${ }^{2}$ Harran University, Faculty of Dentistry, Department of Periodontology, Şanlıurfa, Turkey
}

\section{Correspondence:}

Dr. Abdulsamet TANiK

Adiyaman University, Faculty of

Dentistry, Department of

Periodontology, Adiyaman, Turkey

E-mail:samet.120a@gmail.com

Received: 19 October 2019

Accepted: 13 June 2020

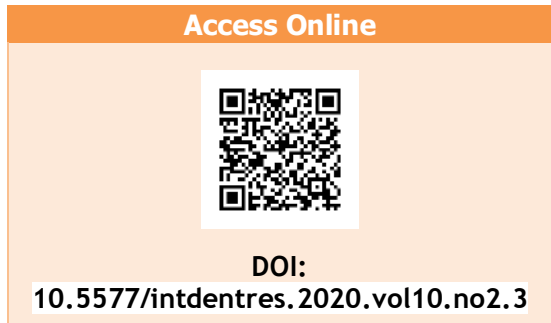

\section{Introduction}

Periodontal diseases are infectious diseases that cause various clinical symptoms in the gums and loss of bone and attachment in supporting tissues. If an inflammatory condition is not controlled, the periodontitis symptoms affecting the supporting tissues develop. Epidemiological studies show that periodontal diseases are among the most common diseases in the world $(1,2)$. The indexes are needed to objectively identify and diagnose the clinical signs of periodontal diseases, to quantify the prevalence and severity of the disease in society, and to determine the degree of destruction of supporting tissues. The main purpose of periodontal indexes is to classify patients with or without periodontal disease based on the numerical values in the indexes. Each periodontal index created to date has its own advantages and disadvantages (2). 
An Ideal Periodontal Index System should be simple, reliable, objective, repeatable and easy to use $(1,3)$.

There are many indexes that cover the progression and etiology of periodontal disease and are used by clinicians to describe changes in oral tissues (2). One of these indexes is CPITN, designed for epidemiological studies. This index was developed by Ainaino et al in 1982. (4). The index, adopted by the World Health Organization (WHO), was modified twice in 1987 and 1997. In addition, one of the two versions of this index is partial CPITN, where 10 teeth are evaluated, and the other is full-mouth CPITN, where 28 teeth are evaluated. (5)

The CPITN index has some limitations. One of them is that because the CPITN index is based on a gradual scoring, pocket measurement is correctly done in approximately $30 \%$ of patients with excessive dental stones and $25 \%$ of patients with gum bleeding and deep pockets. The second is the lack of measurement of tooth mobility and attachment loss, which are important clinical symptoms of periodontal disease. According to these results, the CPITN index, which is widely used worldwide cannot exactly measure periodontal disease (6).

There is a lack of a standard diagnostic method in comparing the studies with CPITN. The aim of this study was to evaluate the sensitivity and specificity of the PCPITN and FCPITN indexes in estimating the diagnosis of periodontal disease.

\section{Materials and Methods}

\section{Sampling}

This study is cross-sectional epidemiological research and was conducted with the permission of the Ethics Committee of Adiyaman University Faculty of Medicine. The study group was created in a total of 1,000 patients, 483 of whom were women and 517 men, who were randomly selected between December 2018 and October 2019. Prior to the application, the objectives of the research were explained to the patients and written informed consent forms were obtained by informing that the participation in the research was voluntary. This study was conducted in patients admitted to the Periodontology Clinic of Adıyaman University Faculty of Dentistry

\section{Clinical oral examination}

In this study, clinical examinations and periodontal measurements of patients were performed by 2 competent periodontists (X.X) using Who sonde (Hu Friedy, Chicago, USA). Ful-mouth periodontal examinations were performed. Individuals with gum bleeding, inflammation and edema in at least one region were evaluated as gingivitis, individuals with more than $3 \mathrm{~mm}$ pocket depth, clinical attachment loss and gingival recession in at least one region were evaluated as periodontitis. Then, diagnostic tests of CPITN versions were compared with sensitivity, specificity and accuracy ratios. A pilot study was conducted to standardize examinations and clinical measurements. Then each dentist examined 20 people in the pilot study and made clinical measurements. Then the examination results of the dentists were compared and the sensitivity and specificity levels among the dentists were calibrated at the ratio of least $80 \%(7)$.

\section{CPITN measurements}

In our research, full-mouth examination of individuals was performed with 4 surfaces of each tooth, and CPITN index data were recorded. In the CPITN index, the presence of healthy periodontium (score 0 ), the presence of gum bleeding (score 1), the presence of tooth stones (score 2), the presence of 4-5 $\mathrm{mm}$ periodontal pockets (score 3 ) and the presence of periodontal pockets (score 4) of $6 \mathrm{~mm}$ and above were determined. The teeth in the posterior region were scored in pairs and the highest value was recorded. Individuals who did not have at least one permanent tooth in each region were not be included in the study. In our study, patients with CPITN 1 and 2 scores were determined as gingivitis, and patients with CPITN 3 and 4 scores were determined as periodontitis group (8). The partial and full version procedures of CPITN index were defined by Ainamo et al. (9).

Partial CPITN index (P CPITN): Ten permanent teeth $(17.16,11,26,27,36,37,31,46,47)$ were examined in the evaluation of existing regions (sextants) scores.

Full-mouth CPITN index (F CPITN): Permanent teeth $(17-14,13-23,24-27,37-34,33-43$, and 44-47) in every region of the mouth except the third molar were examined.

\section{Statistical Analysis}

Data were evaluated in the computer environment with SPSS 23.0 package software (SPSS, Chicago, IL, USA). Simple descriptive mean values are given as standard deviation (SD) and percentage distributions. The ROC curve was created to evaluate sensitivity, specificity, predictive values, diagnostic accuracy rates shown in diagnostic estimation tables. The statistical significance level was considered as $\mathrm{p}<0.05$.

\section{Results}

The distribution of gender, mean age and periodontal status of the patients included in the study groups are shown in Table 1 . One thousand patients with an average age of $32.27 \pm 12.03$, i.e. $517(51.7 \%)$ males and 483 (48.3\%) females, participated in the 
study. The participants had a prevalence of periodontal disease as $94.7 \%$ (gold standard)

Sensitivity, specificity and diagnostic accuracy rates of periodontal screening tests are shown in Table 2. All measured percentage values are made in the $95 \%$ $\mathrm{Cl}$ (confidence range). The measured gingivitis PCPITN sensitivity was $68.88 \%$, the specificity was $84.94 \%$, and gingivitis accuracy was $80.3 \%$. Measured periodontitis FCPITN sensitivity was $89.28 \%$, the specificity was 96.56\% and gingivitis accuracy was $93.1 \%$.

ROC curves of both CPITN versions for gingivitis are shown in Figure 1. Areas below the ROC curve of FCPITN and PCPITN were found to be similar and statistically significant $(p<0.001)$. FCPITN, however, performed slightly better than PCPITN.

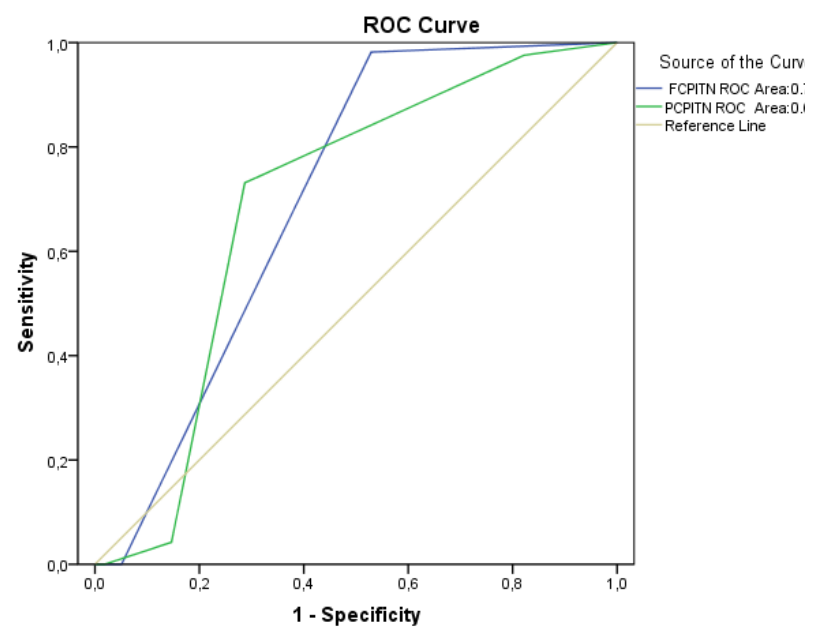

Figure 1. ROC curve for PCPITN and FCPITN in patients with gingivitis

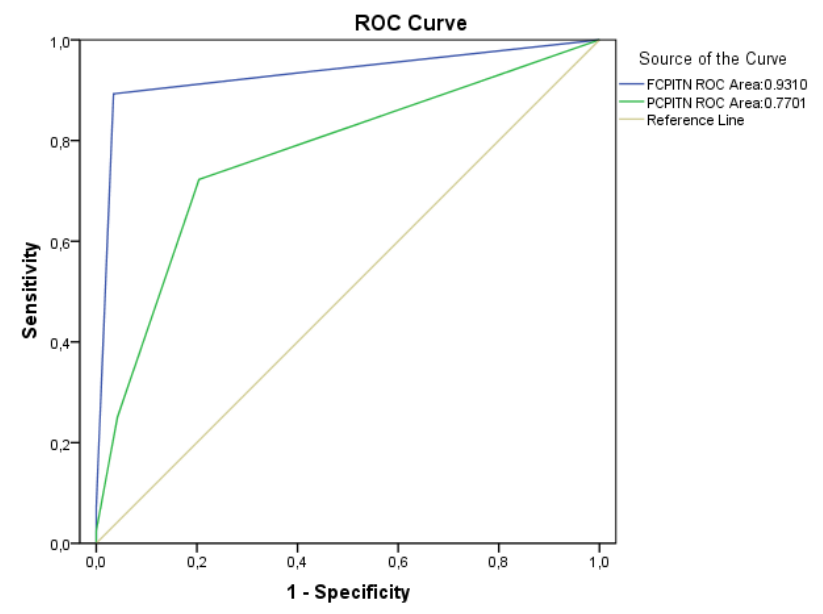

Figure 2. ROC curve for PCPITN and FCPITN in patients with periodontitis

As shown in Figure 2, the ROC curves of both CPITN versions of periodontitis are seen. FCPITN has better area below the ROC curve than PCPITN. It was found to be statistically significant $(p<0.001)$.

All these assessments are based on the largest area under the ROC curve. The best sensitivity and specificity values at cut off points were seen in PCPITN for gingivitis and FCPITN for periodontitis.

Table 1. Demographic characteristics of patients

\begin{tabular}{|c|c|c|c|}
\hline Variable & Categories(N) & Percent(\%) & Mean \pm SD* \\
\hline \multicolumn{4}{|l|}{ Gender } \\
\hline Female & 483 & 48.3 & \\
\hline Male & 517 & 51.7 & \\
\hline Age & & & $32.27 \pm 12.03$ \\
\hline \multicolumn{4}{|c|}{ Periodontal condition } \\
\hline Periodontal health & 53 & 5.3 & \\
\hline Gingivitis & 467 & 46.7 & \\
\hline Periodontitis & 490 & 49 & \\
\hline
\end{tabular}

*Standard Deviation. 
Table 2. Diagnostic power of periodontal disease by PCPITN and FCPITN inde

\begin{tabular}{lcccc|}
\hline & \multicolumn{2}{c}{ Gingivitis } & \multicolumn{2}{c|}{ Periodontitis } \\
\cline { 2 - 5 } Measure & FCPITN(\%) & PCPITN(\%) & FCPITN(\%) & PCPITN(\%) \\
\hline Sensitivity & 34.74 & 68.88 & 89.28 & 25 \\
\hline Specificity & 82.95 & 85.94 & 96.56 & 95.80 \\
\hline Positive predictive value & 50.21 & 70.81 & 95.93 & 84.39 \\
\hline Negative predictive value & 71.98 & 84.81 & 90.84 & 58.44 \\
\hline Diagnostic accuracy & 67 & 80.3 & 93.1 & 62.1 \\
\hline
\end{tabular}

\section{Discussion}

Many periodontal indexes are used in epidemiological studies of periodontal diseases. CPITN index is widely used worldwide today. This is easy to use, fast and cost-effective, but also has several disadvantages. PCPITN index version usually gives biased estimates of the severity and prevalence of deep periodontal pockets and advanced periodontal diseases in the CPITN population (2). The size of these biased estimates depends on the dental groups, some parts of the mouth, and the severity of the disease in the particular population $(10,11)$. In this study, it was analyzed using some parameters related to the prevalence and diagnosis of periodontal diseases in adult patients with methods commonly used in epidemiological studies. Partial and full recording systems and full-mouth findings were compared. While both were significant in the diagnosis of gingivitis, complete recording systems were more successful in the diagnosis of periodontitis.

The basic requirement in epidemiological studies is that the exact definition of the disease to be screened must be made. However, there are no standardized criteria for periodontal disease field scans for this purpose. Due to a wide range of clinical features of periodontal diseases, there are difficulties in developing diagnostic tests in epidemiological research: Periodontal pocket depth, which can be probed and from the clinical features that are particularly helpful in diagnosing periodontitis, and variability of threshold values used in clinical attachment loss and alveolar loss $(5,12)$. As a result, the use of partial recording systems that do not reflect full mouth conditions is becoming common.

In previous studies, the rates of periodontitis and gingivitis in our society were found to be $\% 30.5, \% 69.5$ by Eren et al. (13), and \%30.5, \%69.5 by Ertümer et al. (14), while we found as $\% 49, \% 46.7$, respectively. In our study, the high rate of periodontitis may have been due to the wide age range of adult individuals and the fact that it was made in a narrow area.
In CPITN index, gum bleeding and tooth stone, and periodontal pocket depth are taken as reference for the diagnosis of gingivitis and periodontitis, respectively. In our study, the area below the ROC curve of FCPITN is greater than 0.70 , which suggests that FCPITN is moderately effective in the diagnosis of gingivitis. However, low sensitivity (34.74\%) and high false-negative values may not be suitable for diagnosis. Although it has a high specificity $(82.95 \%)$, the highest sensitivity is desired for screening purposes to determine the disease rather than accidentally showing the disease. The balance between the sensitivity and specificity is determined when the cutoff point is taken as moderate.

PCPITN and FCPITN index have values below the ROC curve close to each other in the diagnosis of gingivitis and are effective in moderate diagnosis, which is sourced from that the CPITN index ignores false pockets, clinical attachment, and alveolar bone loss. (15). Also in our study, the area values under the ROC curve are higher than Taiwo et al. (16) and consistent with the values of Bassani et al. (5).

Although the FCPITN index version used in our study examined all the teeth in the mouth, it was not more effective in the diagnosis of periodontitis due to the choice of the highest in scoring and not taking into account the loss of clinical attachment. As Baelum et al. (17) indicated, the ratio of deep periodontal pockets was found to be low and faulty. Therefore, they reported that the CPITN index has no universal validity.

In our study, the FCPITN index we used in the diagnosis of periodontitis was highly effective with a high ROC value $(93.1 \%)$, high sensitivity $(89.28 \%)$ and high specificity $(96.56 \%)$. These findings are close to the sensitivity value (97.6\%) of Marchoda et al. (10). In contrast, the PCPITN index is moderate effective with a lower ROC and sensitivity value. Also in our study, the area under the ROC curve was found higher than the values of Bassani et al. (5) Its reason can be explained by the large sample volume of our study and the different heterogeneity of the population. It can also be thought that the reason is that the ROC curve has different cutoff points. In this study, the lower values 
of the PCPITN version under the ROC curve may be due to the evaluation of data from only 10 teeth in the mouth by the PCPITN index. As mentioned in previous studies, the diagnosis of severe periodontitis is more valuable than chronic periodontitis for CPITN index $(5,9)$. When studying with the CPITN index, it is necessary to be cautious in interpreting the CPITN 's result data carefully, especially in population selection.

\section{Conclusions}

CPITN periodontal index is still widely used worldwide, although it has some disadvantages. Although FCPITN and PCPITN indexes, which are CPITN index versions, were close to being moderate effective in gingivitis diagnosis, they were found to be more effective in periodontitis diagnosis. We think that FCPITN index is particularly effective in diagnosis of periodontitis at an excellent level. In order to achieve a more accurate result in the diagnosis of periodontal diseases by these two versions, epidemiological studies with large sample volumes are needed.

Ethical Approval: Ethics committee approval was received for this study from Adlyaman University, Faculty of Medicine Ethics Committee in accordance the World Medical Association Declaration of Helsinki, Ethics Committee in accordance the World Medical Association Declaration of Helsinki, with the approval number: 2018/8_2.

Peer-review: Externally peer-reviewed.

Author Contributions: Conception - A.T.; Design - A.T., M.G.; Supervision - A.T.; Materials - M.G.; Data Collection and/or Processing - A.T., M.G.; Analysis and/or Interpretation - A.T.; Literature Review A.T., M.G.; Writer - A.T., M.G.; Critical Review - A.T.

Conflict of Interest: No conflict of interest was declared by the authors.

Financial Disclosure: The authors declared that this study has received no financial support.

\section{References}

1. Newman MG, Takei HH, Klokkevold PR, Carranza, F.A. Glickman's clinical periodontology, 10 th ed. Beck JD, Arbes SJ.Chapter 8: Epidemiology of Gingival and Periodontal Diseases.China, Elsevier Saunders 2007;110-131.
2. Dhingra K, \& Vandana KL. Indices for measuring periodontitis: a literature review. International dental journal, 2011;61(2):76-84. (Crossref)

3. Carter HG \& Barnes GP. The gingival bleeding index. Journal of Periodontology, 1974;45(11):801-805. (Crossref)

4. Ainamo J, Barmes, D, Beagrie G, Cutress T, Martin J\&SardoInfirri, J. Development of the World Health Organization (WHO) community periodontal index of treatment needs (CPITN), International Dental Journal 1982;32:281-291.

5. Bassani DG, Silva CMD, \& Oppermann RV. Validity of the Community Periodontal Index of Treatment Needs'(CPITN) for population periodontitis screening. Cadernos de saude publica, 2006;22:277-283. (Crossref)

6. Benigeri M, Brodeur JM, Payette $M$, Charbonneau A \& Ismaïl A. I. Community periodontal index of treatment needs and prevalence of periodontal conditions. Journal of clinical periodontology. 2000;27(5):308-312. (Crossref)

7. Pine CM, Pitts NB, Nugent ZJ. BASCD guidance on the statistical aspects training and calibration of examiners for surveys of child dental health. A BASCD coordinated dental epidemiology programme quality standard. Comm Dent Health 1997;14(sup 1):18-29.

8. Kesim S, Unalan D, Esen C, et al. The relationship between periodontal disease severity and state-trait anxiety level. J Pak Med Assoc, 2012;62:1304-8.

9. Ainamo J, Ainamo A. Partial indices as indicators of the severity and prevalence of periodontal disease. Int Dent J 1985;35:3226.

10. Machado V, Botelho J, Mascarenhas P, Cavacas MA, Alves R \& Mendes JJ. Partial recording protocols performance on the assessment of periodontitis severity and extent: bias magnitudes, sensibility, and specificity. Revista Portuguesa De Estomatologia Medicina Dentaria E Cirurgia Maxilofacial, 2018;59(3):145-153. (Crossref)

11. Kingman A, Susin C, Albandar JM. Effect of partial recording protocols on severity estimates of periodontal disease. J Clin Periodontol. 2008;35:659-67. (Crossref)

12. Jenkins WM\&Papapanou PN. Epidemiology of periodontal disease in children and adolescents. Periodontology 2000. 2001;26(1):16-32. (Crossref)

13. Eren, Gülnihal, and Sema Becerik. "Evaluating the Periodontal Status and Oral Hygiene Habits Among Dental Patients." Istanbul Medical Journal. 2016;17(3):93-98. (Crossref)

14. Ertüme, Ezgi, Fatma Karacaoğlu, and Murat Akkaya. "Ankara Üniversitesi Diș Hekimliği Fakültesi'ne başvuran hastaların periodontal hastalık öz bildirim geçerliliğinin değerlendirilmesi." Selcuk Dental Journal 2018;5(1):39-49. (Crossref)

15. Kingman $\mathrm{A}$, Loe $\mathrm{H}$, Anerud $\mathrm{A}$, Boysen $\mathrm{H}$. Errors in measuring parameters associated with periodontal health and disease. $J$ Periodontol 1991;62:477-86. (Crossref)

16. Taiwo JO, Ibiyemi O, Osuh ME \& Lawal FB. Validity of the periodontal disease surveillance self-report questionnaire in a Nigerian population. Port Harcourt Medical Journal. 2017;11(2):84. (Crossref)

17. Baelum, V., \& Papapanou, P. N. CPITN and the epidemiology of periodontal disease Commentary. Community dentistry and oral epidemiology. 1996;24(6):367-368. (Crossref) 